\title{
A digital encoder for field recording of behavioral, temporal, and spatial information in directly computer-accessible form
}

\author{
CLIFTON LEE GASS \\ Department of Zoology, University of British Columbia, Vancouver, British Columbia, Canada V6T 1W5
}

Digitorg is an inexpensive, portable, field-operable event recorder for recording both behavioral and spatial information in real-time on audiotape. Its capacity is 256 codes. Behavioral information is recorded by pressing buttons on a keyboard and spatial information is recorded by touching a magnetic wand to a paper map overlying a matrix of switches. The audiotape recordings are played through a simple interface into a small computer, producing a permanent record of the time and identity of each event. The record is then available for analysis. Digitorg is discussed in relation to the general value of analyzing spatially differentiated behavior sequences.

Because of the difficulties of recording and analyzing spatially differentiated behavior sequences by conventional methods, it is tempting to treat spatial aspects superficially or to ignore them altogether. But some phenomena are essentially spatial in nature, such as flocking, schooling, foraging, and exploratory behavior. In addition, social hierarchies and social interactions in general, as well as many other phenomena, can be strongly affected by spatial factors. This paper describes a method for recording behavioral and spatial information in real-time.

Any encoding system with sufficient capacity can code locations if its keyboard is designed properly. A system can be used to code sequences according to other factors as well, such as the array of items in a supermarket, the individuals in a group, and so forth. The theoretical context of the present author's recording requirements are described here, in order to emphasize the value of spatial coding.

The hummingbirds studied in the present author's research are territorial: Each individual defends and forages within an area of two-dimensional habitat. The flowers in the territory produce nectar whose total caloric value approximates the caloric needs of the bird (Gass, Angehr, \& Centa, 1976). Each flower is fixed in space, but its nectar supply is renewable at a known

Fred Attneave suggested the idea of an encoder capable of recording spatial information. Lee Vernon designed an early analog model, which the present author built, using funds from the University of Oregon Biology Department. Digitorg was designed by Steve Borden, who was also a constant source of encouragement throughout the project. Rob Knight, Fleet Berry, and Dave Holmes provided technical advice and assistance. Robin Liley, Sarah Groves, Steve Borden, and four anonymous reviewers read and commented on the manuscript. The project was supported by Grants 67-5098 and 67-5134 from the University of British Columbia and Grant 67-9876 from the National Research Council of Canada. rate. The energetic profitability of visiting a flower for nectar depends on its location in relation to the bird, its nectar production rate, and the time since it was last visited. Time between visits depends on the food requirements of the bird, the number and type of flowers in the territory, and the spatial patterning of visits among the flowers. Individuals with "good" territories spend nearly all the time visible and within their territories. The distribution of behavior is recorded as it occurs at the perches and patches of flowers within individual territories. With this information, the spatial distributions of foraging behavior and resource availability can be studied as they develop in relation to each other, and a number of predictions from theory can be tested.

\section{GENERAL DESCRIPTION OF THE SYSTEM}

Digitorg (Digital Behavior Organ) is a portable fieldoperable system that produces a coded record on audiotape of the temporal distribution of switch closures. Digitorg cannot encode simultaneously occurring events, because the system is insensitive for about $70 \mathrm{msec}$ after each switch closure. Because 256 different software-defined codes are available; most can be reserved as a coordinate system for encoding spatial information. The record approaches $.1 \mathrm{sec}$ in temporal resolution and is directly computer-accessible, eliminating hand recording or processing of data. Using Dawkins' (1971) system, Halliday (1975) illustrated the power of this general approach in a laboratory study of temporal patterning of mating behavior in newts. Digitorg is similar in some respects to several other systems recently developed (Beauchamp \& Scobie, 1973; Dawkins, 1971; Fernald \& Heinecke, 1974; Lentz \& Haith, 1969; McPartland, Foster, \& Kupfer, 1976; Stephenson, Smith, \& Roberts, 1976; White, 1971). Of these, only Dawkins' and Stephenson's 
Table 1

Comparison of Three Field-Operable Encoding Systems

\begin{tabular}{lccc}
\hline & Dawkins (1971) & Stephenson (1976) & Digitorg \\
\hline Capacity (number of codes) & 100 & 48 & 256 \\
Encodes durations directly? & no & yes & no \\
Encodes simultaneous events? & no & yes & no \\
Temporal resolution (seconds) & .02 & .05 & no \\
Designed to encode spatial in formation? & no & yes & no \\
Redundant recording & no & time-multiplexed digital & serialized digital \\
Encoding method & analog (frequency) &
\end{tabular}

systems and Digitorg are designed for field use. Table 1 compares the three systems.

Signals from switches representing behaviors and locations are digitized and serialized by a diode matrix and CMOS logic circuits, and are then used to gate an audio oscillator for digital recording in binary code on a standard or stereo tape recorder (Figure 1). Voice may be recorded simultaneously with the stereo recorder. Decoding is done automatically by playing the audiotapes at four times the recording speed through an interface into a PDP-11 or other small computer that can be employed in real-time. The computer produces a file on magnetic tape, consisting of a pair of data words for each record on audiotape. One word is a code representing the identity of a switch and the other is the time that the switch closed.

The heart of Digitorg is its logic module, which is identical in all applications. Although few users will need the full 256-code capacity of the module, no significant savings are gained by designing it for lower capacity.

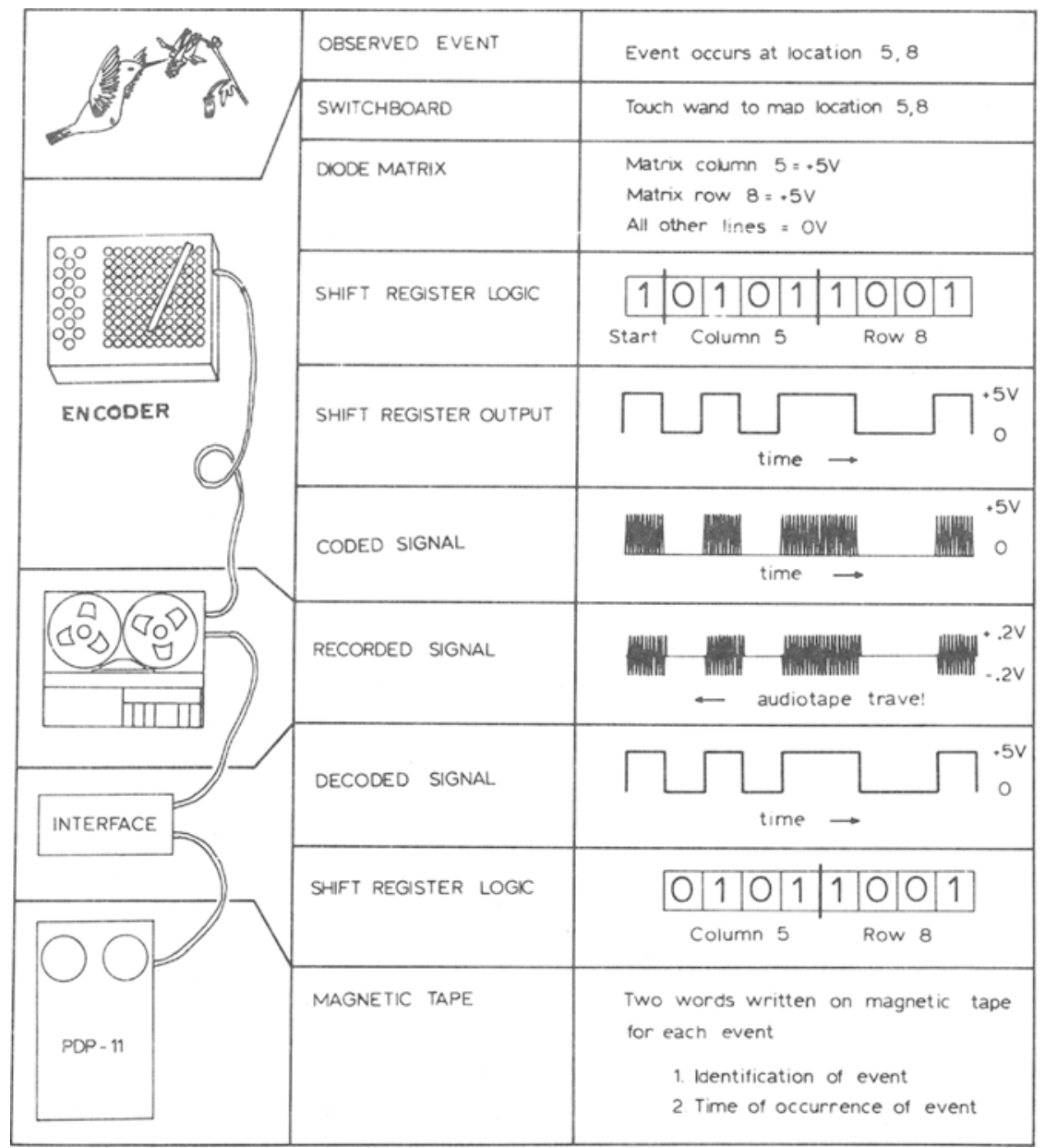

Figure 1. Information processing by Digitorg. Stages of infurmation processing are shown from top to bottom. 
The module consists of 31 electronic components, mounted on a $12.1 \times 13.3 \mathrm{~cm}$ glass circuit board. It can be assembled and wirewrapped in 1 day by an inexperienced person. The number of switching devices determines the capacity of Digitorg, and their type and spatial arrangement largely determines its cost.

In the field encoder, 14 switches code behaviors, 100 code locations, and 1 (SYSBUT) communicates with the computer. In laboratory applications, the same logic circuitry is operated automatically by 37 phototransistors that sense a hummingbird's bill at one of an array of feeders or its body at a perch. Cost of parts for the logic module is less than $\$ 50$ Canadian. Total cost of parts for the field encoder is about $\$ 500$; the laboratory encoder is about $\$ 400$. Cost of parts for the decoder and computer interface is about $\$ 100$.

Important considerations for the tape recorder are maximum length of recording session, recording and playback speed, and stability of tape speed. A' stereo recorder is required if simultaneous recording of voice information and encoder output is desired (this is highly recommended). Digitorg has been used successfully with a Uher stereo quarter-track 5-in. reel-to-reel recorder (4400 Report Stereo IC) in continuous 4-h recording sessions at $2.4 \mathrm{~cm} / \mathrm{sec}$ on $1,200-\mathrm{ft}$ tapes.

Encoding is insensitive to temperature or to long-term $P .1 \mathrm{sec}$ ) fluctuations in tape speed. However, timing is estimated by the computer from placement of codes along the audiotape, so any long-term fluctuations in tape speed affect estimated times of occurrence and durations of events. Although these errors are later corrected (see below), the recorder should be buffered against rapid changes in outdoor air temperature by placing it in the shadows; batteries should be fresh. One Globe Gel-Cell rechargeable storage battery (Globe Battery Division, Globe Union Co., 900 E. Keefe Ave., Milwaukee, Wisconsin; GC $1200 ; 3.2 \mathrm{~kg}$ ) will operate the Uher recorder for $30 \mathrm{~h}$.

\section{THE DIGITORG FIELD ENCODER: CONSTRUCTION AND USES}

After being transported to remote field locations by mule and backpack, and after being dropped, rained on, and frozen, Digitorg has operated successfully for $216 \mathrm{~h}$; the system is readily adapted to other specific encoding needs. But Digitorg requires adequate support, including proper computing facilities, programming capability, and competent electronic consultation.

\section{Encoding}

The field encoder is housed in an aluminum box, $18 \times 28 \times 8 \mathrm{~cm}$ in size, and weighs $1.8 \mathrm{~kg}$. The unit has 15 normally open pushbutton switches (Alcoswitch Co., Lawrence, Massachusetts; No. MSPM 101C) mounted in three columns on the left of the box (Figures 1 and 2), which represent behaviors being observed. The observer can record with one hand, encoding the beginning and/or end of specific behaviors, with durations being calculated later by the computer. At the right of the pushbuttons are 100 normally open magnetically operated switches (Fifth Dimension Corporation, P.O. Box 483, Princeton, N.J.; No. LC2), mounted in a 10 by 10 matrix on $1 / 2$-in. centers under the lid of the box (Figures 1 and 2). On top of the lid and overlying the switch matrix is a paper map representing locations. The observer "plays" the location switches by probing the map with a magnetic wand held in the other hand. An indented Plexiglas "antiambiguator," mounted over the map, centers the wand over the proper map location and switch, reducing the possibility of encoding errors (Figure 2).

The paper map is a sheet of light cardboard, printed with a 10 by 10 array of black dots on $1 / 2$-in. centers. Switches to be used during a recording session are identified by coloring $1 / 2$-in. circles around the appropriate dots with felt-tip pens. The antiambiguator is made by boring 3/4-in. depressions into 1/4-in. clear Plexiglas, and then carefully fire-polishing to restore transparency. The magnetic wand is a cylinder of $3 / 8$-in. diam Alnico 8 magnet, ground without heating to the proper length, and rounded to fit into the antiambiguator depressions. The magnet is too long if any switch closes when the wand rests anywhere on the ridges between depressions; it is too short if any switch fails to close when the wand rests at the bottom of the depression over it.

The computer is instructed to associate all behavior codes that follow a location code with that location. The observer encodes a series of behaviors that occur in a given location by a single probe of the appropriate location on the map, followed by a series of presses of the appropriate behavior buttons. With practice, simple encoding requires no conscious logical processing of either behavioral or spatial information. This method provides a tremendous advantage over conventional methods, because it allows faster processing of more information.

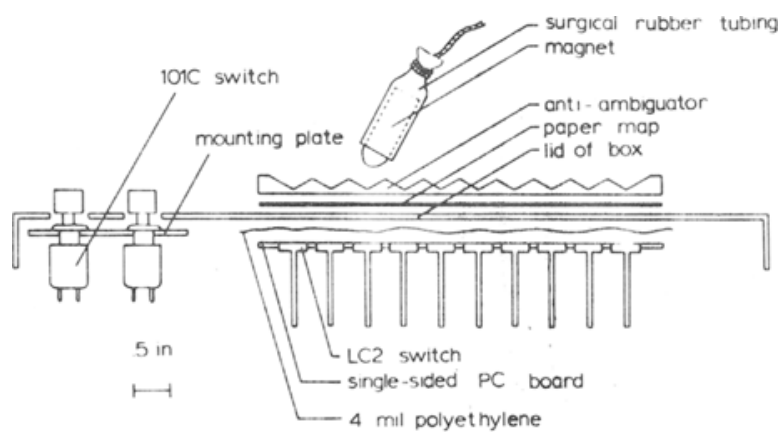

Figure 2. Section through lid of encoder, showing keyboard construction. Both switch assemblies mount tightly against the lid. Details are discussed in the text. 
The map is drawn to "look like" the real world, as it appears from the perspective of the observer, without attempting to conserve real plan relationships. Digitorg only requires clearly visualizing the analog, associating it with the map, and coupling the association with appropriate movements of the hand. Encoding becomes automatic, so that changing to new map areas involves only visualizing and drawing a new analog, and appropriately modifying the coupling between analog, map, and movements of the hand. The observer can move easily between areas; the process of drawing a new map prepares one to use it after only a few minutes' practice. After 1 day's practice, an observer can easily follow hummingbird foraging flights and encode long sequences of behaviors at a rate of up to two per second, and simultaneously record verbal information that either elaborates or provides a broader context for the coded information, such as the concurrent behavior of adjacent individuals.

\section{Communicating with the Computer}

Information flows via the tape recorder from Digitorg to the computer. By using a somewhat general and flexible program, some of the instructions can be supplied by Digitorg along with the data. This allows the observer to control the processing of each data set and to adapt the information processing system to the particular requirements of each observation session. Possible uses of encoding options are varied and subject only to the operator's ability to encode reliably and to design appropriate computer programs.

One arbitrarily selected pushbutton (SYSBUT) instructs the computer how to process information. All other buttons simply encode data. Messages for the computer are encoded as numbers of presses of SYSBUT in quick succession, as shown below:

One press. Mistake; replace the last code with the next, retaining the time of the former.

Two presses. Mistake; replace the last two codes with the next two, retaining the times of the former. Most mistakes in encoding are noticed immediately, through visual or kinesthetic feedback. Use of SYSBUT automatically corrects faulty encoding, without sacrificing correct timing.

Three presses. Voice information to follow on the other audio track; file the time and calculate the tape footage. Use of SYSBUT allows automatic compilation of a list of times and tape footages at which voice information is to be found. This facilitates transcription and makes possible integration of the vocal and encoded information.

Five presses. Pause/resume; do not include time between these marks in the record. For short pauses, the tape recorder is left running and the pause is automatically accounted for.

Seven presses. Timing calibration.

Nine presses. Begin/end session. Errors in timing are minimized by keeping written records of the time of beginning and end of each recording session, as well as of timing calibration codes repeated at $10-$ to $20-\mathrm{min}$ intervals. These standards are then used by the computer as a basis for recalculating all timing estimates for the session. Files of field data are typically in error less than 3\% before correction. For applications demanding particularly great temporal accuracy timing calibration codes could be repeated as often as necessary, and could be generated automatically if a buffering or priority system were added to the circuitry.

\section{Digitizing, Serializing, and Recording}

Digitization of output from the encoder switches occurs in two stages. In the first stage, signals from switches are translated into unique pairs of signals by a 16 by 16 diode matrix. In the second stage, signals on 32 parallel lines from the diode matrix lead to CMOS logic circuits, which translate the pairs of signals into binary representations of position within the diode matrix (Figure 3).

The binary code word identifying a switch closure is 9 bits long. The code word consists of three parts: a start bit (always on) and two 4-bit numbers identifying the column and row of the switch within the diode matrix. Before the binary code word can be recorded on one track of audiotape, it must be serialized by translating it from the form of simultaneous voltage levels in parallel lines into the form of a temporal sequence of voltage levels on a single line. This is done by loading the 8 data bits into a shift register, shifting it at regular intervals, and then using the serial sequence of voltage levels to gate an audio oscillator that outputs $2,160 \mathrm{~Hz}$ to the recorder. A positive voltage in the gating line results in a short $(7.4-\mathrm{msec})$ burst of $2,160 \mathrm{~Hz}$ being recorded on audiotape. Thus, the 8-bit binary code word is represented on audiotape by a single start burst of $7.4 \mathrm{msec}$, followed by eight 7.4-msec segments that may contain audio signals, depending on which switch was closed.

The circuitry in the upper left of Figure 3 digitizes the information; the rest of the circuitry serializes it and produces the audio signal. Digitization, serialization, and recording are completed $67 \mathrm{msec}$ after switch closure, and the system is then enabled for another switch closure.

\section{Decoding and Interfacing to the Computer}

A variety of decoding options are possible, depending on the computer installation. The decoder used by the present author uses TTL integrated circuits and is permanently installed in the PDP-11/45. With modifications, it can be used with any Digital "family-of-8" computer, or with any computer that uses a priority interrupt system. Because the present author's PDP-11 runs under the UNIX time-sharing operating system, however, the decoding software is specific to his installation. The following section describes the decoding process in general terms. Requests for assistance with 

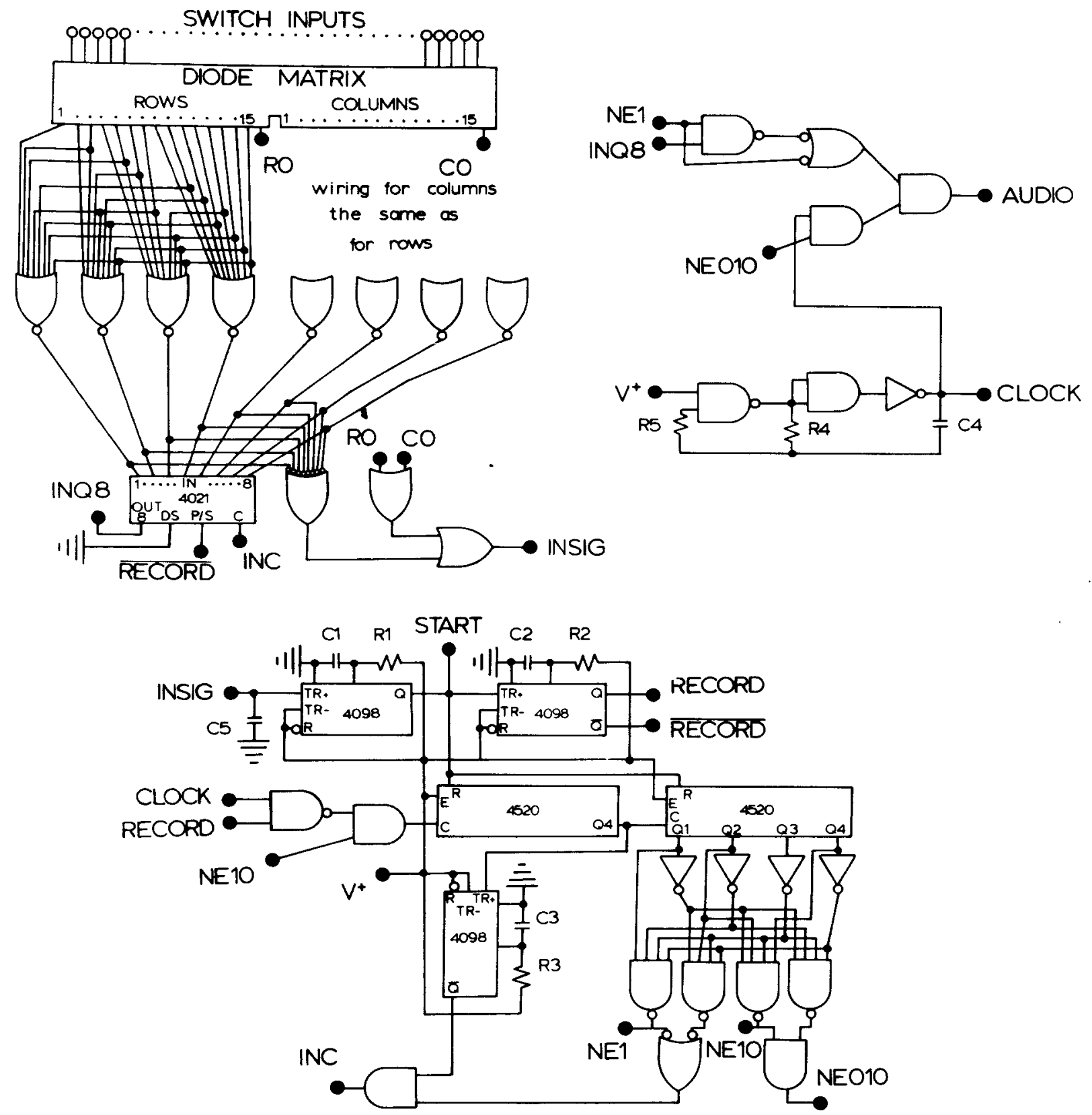

Figure 3. Digitorg logic module circuitry. RCA CMOS integrated circuits are listed in Table 2, and resistor and capacitor values are listed in Table 3. Power is $+9 \mathrm{~V}$ (Eveready No. 1222). A two-input AND gate was omitted from Figure 3 . Inputs: INSIG and RECORD. Output: TR+ on dual monostable 4098-1. No additional components are required.

decoding problems, or other problems, should be directed to the author.

Tapes that were recorded at $2.4 \mathrm{~cm} / \mathrm{sec}$ are played through a decoding interface at $9.6 \mathrm{~cm} / \mathrm{sec}$ into the computer. Data bits in the form of short bursts (16 cycles) of $2,160 \mathrm{~Hz}$, serially recorded on audiotape, are demodulated and amplified, loaded into an 8-bit shift register, then read by the computer as one 8-bit byte.

The incoming audio signal from the tape recorder is first converted to pulses that resemble the original unrecorded Digitorg output. When the first pulse arrives, a counter starts counting the output of a high-frequency clock. When the ninth pulse arrives (halfway through the start bit), the counter is stopped, the count is doubled (to equal the duration of 1 bit), and another counter starts counting the output of the clock. When the two clock counters are equal (halfway through the first data bit), one of them is reset and restarted and the value $(0$ or $+5 \mathrm{~V})$ of the first demodulated bit is loaded into a shift register. This is repeated until 8 bits have been loaded, then an interrupt flag to the computer is raised and the 8-bit byte is read into a buffer along with the contents of the computer's real-time clock. 
The buffer, when full, is written into a file on the disk. At this stage, the codes in the file are converted into symbols understandable to the user by means of a translation table. In the present author's field application, codes are four-letter symbols for behaviors (perch = PRCH) and four-digit decimal coordinates for locations (Column 5, Row $8=0508$ ). The file is available for transfer to magnetic tape or for printing.

Correction of estimated times of occurrence of events is done later by a separate program. This program compares estimated times of occurrence of timing calibrations (7 SYSBUT) with the actual times that were hand recorded in the field, calculates a correction factor, then recalculates all estimated times by linear interpolation between timing calibrations. Corrected files are then available for analysis.

\section{Analysis}

An important feature of Digitorg is that files collected with it are sequential files of "instantaneous" events. These represent transitions between states of the system being observed, such as the transition between perching and flying. Higher order events, usually the ones of interest, must be constructed contextually from the sequences of instantaneous events. For example, a file might contain the sequence: ..... PRCH, 0203, OFP, $0508, \ldots \ldots$, PRCH, $0203 \ldots$. , indicating that the hummingbird perched at Location 2,3, left the perch, arrived at Location 5,8, performed a sequence of behaviors, then returned to the same perch, all at specific times. Of importance is the proportion of time spent performing various behaviors or behaving at various locations. For instance, the event "perch" began at the first code PRCH and ended at OFP, and the event "flight" began at OFP and ended at the second code PRCH. Perch and flight are mutually exclusive, but each could contain a number of lower order events. Depending on what happened at Location 5,8, flight might be a foraging, chasing, or some other type of flight. The possibilities are extensive. Files containing higher order events, their times, and their durations are constructed by special programs.

Table 2

List of CMOS Integrated Circuits

\begin{tabular}{lcc}
\hline \multicolumn{1}{c}{ Function } & Device & $\begin{array}{c}\text { Number } \\
\text { of ICs }\end{array}$ \\
\hline INVERTER & 4049 & 1 \\
OR & 4071 & 1 \\
2-input AND & 4081 & 2 \\
2-input NAND & 4011 & 1 \\
4-input NAND & 4012 & 2 \\
8-input NOR & 4078 & 8 \\
8-input NAND & 4068 & 1 \\
dual monostable & 4098 & 2 \\
8-stage static shift register & 4021 & 1 \\
dual binary counter & 4520 & 1 \\
\hline
\end{tabular}

Table 3

List of Timing Circuits

\begin{tabular}{ccc}
\hline Component & $\begin{array}{c}\text { Nominal } \\
\text { Value of } \\
\text { Component }\end{array}$ & $\begin{array}{c}\text { Measured } \\
\text { Time Constant }\end{array}$ \\
\hline R1 & $820 \mathrm{~K}$ & $110 \mu \mathrm{sec}$ \\
C1 & $100 \mathrm{pf}$ & \\
R2 & $470 \mathrm{~K}$ & $70 \mathrm{msec}$ \\
C2 & $.47 \mathrm{fd}$ & $110 \mu \mathrm{sec}$ \\
R3 & $820 \mathrm{~K}$ & \\
C3 & $100 \mathrm{pf}$ & $380 \mu \mathrm{sec}$ \\
R4 & $110 \mathrm{~K}$ & \\
R5 & $470 \mathrm{~K}$ & $10 \mu \mathrm{sec}$ \\
C4 & $1500 \mathrm{pf}$ & \\
C5 & $.1 \mu \mathrm{f}$ & \\
\hline
\end{tabular}

Note-Circuits are discussed in detail in the RCA applications notes for the devices used.

\section{Example of Rough Analysis of a Field-Data File}

In one recording session (August 15, 1975; File F6A1-75), 1,818 behavioral events were recorded in $180 \mathrm{~min}$ (estimated duration before correction was $175.14 \mathrm{~min}$ ). The observer also entered 24 voice records (3 SYSBUT), entered four timing calibration codes (7 SYSBUT), and corrected six encoding errors (1 SYSBUT).

The resident hummingbird perched 122 times on two perches for a total of $138.85 \mathrm{~min}(77.1 \%$ of time), was gone from the territory nine times for a total of $2.38 \mathrm{~min}(1.3 \%$ of time), and flew within the territory $33.80 \mathrm{~min}$ (18.8\% of time). The bird made 941 visits to flowers in eight patches of one species and 166 visits to flowers in seven patches of another species. It chased other hummingbirds 38 times from 12 patches and 3 times from the vicinity of its own perch. It was chased by other birds 63 times at 12 patches and 10 times from its own perch. It attempted to catch small flying insects 12 times and chased bees twice from two patches of flowers. There were unusually many aggressive interactions during this session. The territory was unusually rich (about twice as many flowers as average), and the bird suffered unusually many foraging intrusions from other individuals, one of whom also repeatedly chased the resident. By the next day, the territory was smaller and there was less aggression.

\section{REFERENCES}

Beauchamp, J., \& Scobie, S. R. A high-speed computercompatible event recorder. Behavior Research Methods \& Instrumentation, 1973, 5, 418-424.

DAwkins, R. A cheap method of recording behavioral events, for direct computer-access. Behaviour, 1971, 40, 162-173.

Fernald, R. D., \& Heinecke, P. A computer compatible multi-purpose event recorder. Behaviour, 1974, 48, 268-275.

Gass, C. L., Angehr, G., \& Centa, J. Regulation of food supply by feeding territoriality in the rufous hummingbird. Canadian Journal of Zoology, 1976, 54, 2046-2054.

Halliday, T, R. An observational and experimental study of 
sexual behaviour in the smooth newt. Triturus vulgaris (Amphibia: Salamandridae). Animal Behaviour, 1975, 23, 291-322.

LENTZ, R., \& HarTh, M. M. Audio tape storage of experimental data: An application to tachistoscope research with children. Behavior Research Methods \& Instrumentation, 1969, 1, 273-275.

McPartland, R. J., Foster, F. G., \& Kupfer, D. J. A computer-compatible multichannel event counting and digital recording system. Behavior Research Methods \& Instrumentation, 1976, 8, 299-301.
Stephenson, G. R., Smith, D. P. B., \& Roberts, T. W. The SSR system: An open-format event recording system with computerized transcription. Behavior Research Methods \& Instrumentation, 1976, 8, 259-277.

WhITE, R. E. C. WRATS: A computer compatible system for automatically recording and transcribing behavioural data. Behaviour, 1971, 40, 135-161.

(Received for publication September 20, 1976; revision accepted November 12, 1976.) 